\title{
Interaction between Free-Surface Oscillation and Bubble Translation in a Megasonic Cleaning Bath
}

\author{
Yu Katano ${ }^{1, a}$ and Keita Ando ${ }^{1, b^{*}}$ \\ ${ }^{1}$ Keio University, 3-14-1 Hiyoshi, Kohoku-ku, Yokohama, Kanagawa 223-8522, Japan \\ akatanoyuu@gmail.com, bkando@mech.keio.ac.jp \\ ${ }^{*}$ Corresponding author
}

Keywords: Megasonic cleaning bath, free-surface oscillation, acoustic cavitation, bubble translation

\begin{abstract}
Visualization experiments were performed to study the relation between free-surface motion and bubble translation in a $1-\mathrm{MHz}$ ultrasonic cleaning bath. From the visualization with a video camera, the characteristic frequencies of the free-surface oscillation (under the acoustic radiation force) and the translational velocity of cavitation bubbles (trapped via the primary Bjerknes force) were extracted, showing that there is a strong correlation between the free-surface oscillation and bubble translation. From the context of megasonic cleaning, such free-surface oscillation is expected to contribute to uniform cleaning performance with cavitation bubbles.
\end{abstract}

\section{Introduction}

Underwater megasonic wave irradiation is used to remove small particles from delicate surfaces such as semiconductor wafers; it is the so-called megasonic cleaning technique [1]. Recent studies on megasonic cleaning suggest that acoustic cavitation serves as the main cleaning agent [2]. Oscillating bubbles can be trapped by the primary Bjerknes force [3] at the antinodes and nodes of the pressure field when a standing-wave-like acoustic field appears in ultrasonic cleaning baths [4]. This gives rise to nonuniform cleaning performance. It is thus favored that acoustic bubbles move around cleaning targets. In addition to these cavitation phenomena, there is an important acoustic phenomenon in ultrasonic cleaning baths; underwater ultrasound irradiation elevates the free surface of the water in due to acoustic radiation force as a result of acoustic impedance discontinuity across the free surface [5]; this effect is emphasized in the case of higher-frequency ultrasound due to its higher directivity [6]. If the free surface exhibits oscillation, the pressure field inside cleaning baths will show periodic unsteadiness, which is expected to avoid trapping of acoustic bubbles at the specific positions. Therefore, it is essential to understand the relation between the free-surface motion and bubble translation in megasonic cleaning baths.

In the present study, we visualize, with a video camera, free surface and bubbles in a $1-\mathrm{MHz}$ ultrasonic cleaning bath. From Fourier analysis of the free-surface motion and bubbles' translation velocity, we discuss their correlation in the context of ultrasonic cleaning.

\section{Experimental Method}

Figure 1(a) shows the setup for visualization of free surface in a megasonic cleaning bath made of transparent acrylic plates. We used a cylindrical-shaped transducer (1Z30I, Japan Probe) which has a hermetically sealed structure in which a cylindrical piezo-electric ceramic of $30 \mathrm{~mm}$ in diameter is embedded in a cylindrical ABS container of $38 \mathrm{~mm}$ in diameter. The transducer was mounted at the bottom of the bath. Air-saturated deionized water was filled in the bath, and undisturbed free surface elevation from the transducer's surface was set at $76 \mathrm{~mm}$. Sinusoidal wave whose frequency is $1 \mathrm{MHz}$ was generated continuously by a multifunction generator (WF1973, NF Co.), magnified by a power amplifier (HSA4014, NF Co.), and fed to the transducer. Output power from the transducer was set at $2.4 \mathrm{~W}$ (calculated based on hydrophone pressure measurement [7]). The water temperature was at $20^{\circ} \mathrm{C}$, so that the corresponding ultrasound wavelength $\lambda$ is $1.48 \mathrm{~mm}$. Note that the water temperature was almost undisturbed during the sonication. 
In order to visualize motion of the free surface, fluorescent dye (rhodamine B) was seeded in the water, and an Nd:YAG CW laser sheet (G100-KA, Kato Koken Co.) was illuminated along the $z$ axis from the top; see the coordinate system depicted in Fig. 1(a). The fluorescence was recorded by a video camera (FASTCAM SA-X2, Photron) through a long-pass filter (VIS O-58 50MM, Edmund optics). Visualization was performed around the center of the free surface, $(x, y, z)=(0,0,0)$, where the elevation is the most significant. The camera pointed down at 30 degrees above the free surface in order to avoid the meniscus on the wall and surface wave in the foreground of the working plane. Figure 1(b) shows an example of the visualized free surface. The recorded images were binarized by Otsu's method, then the free-surface displacement $h(t)$ from the undisturbed free surface along the $z$ axis was calculated. This measurement was done not only during the ultrasound irradiation but also after it was stopped, which allows for calculation of the natural frequency of the free-surface oscillation.

Figure 2(a) shows the setup for visualization of cavitation bubbles inside the megasonic cleaning bath. We visualized cavitation bubbles by a high-speed camera (FASTCAM SA-X2, Photron) with LED back lighting. The visualization spot is around $(x, y, z)=(0,0,-H / 2)$, where cavitation bubbles appear densely and the periodicity of the bubble translation is remarkable. Figure 2(b) shows an example of the visualized cavitation bubbles. We analyzed the translational velocity of individual bubbles from the recorded images by a commercial software (KM2.0, Kato Koken Co.). We calculated the vertical velocity spatially averaged in the visualized area, $W(t)$, as a representative value. Note that the velocity of bubbles whose radius is smaller than $4 R_{r}$ cannot be detected in this image processing. Here, $R_{r}$ represents the resonant bubble radius that is calculated at $3.3 \mu \mathrm{m}$ for 1$\mathrm{MHz}$ pressure forcing [8].

(a)
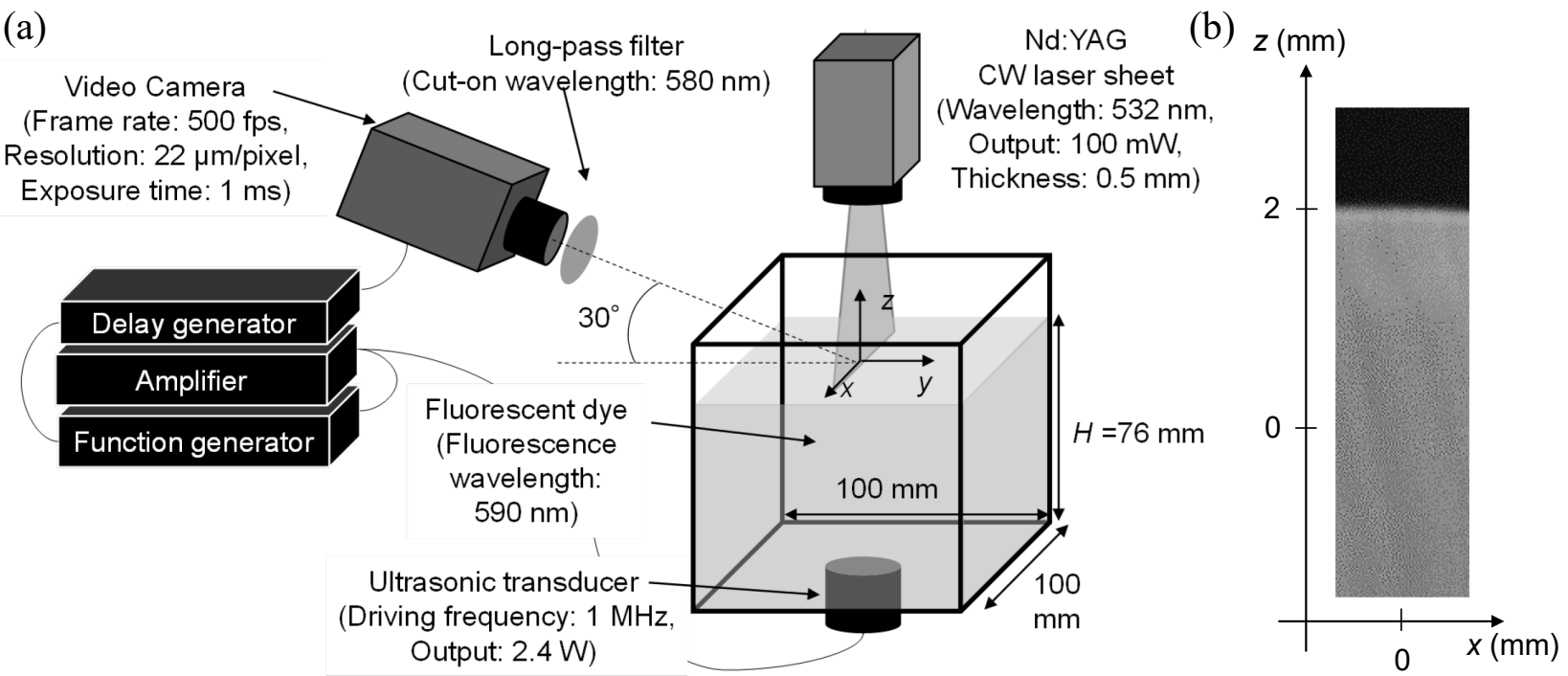

Figure 1. (a) Schematic of the setup for visualization of the free surface in a megasonic cleaning bath. (b) A typical snapshot of the free surface. 
(a)

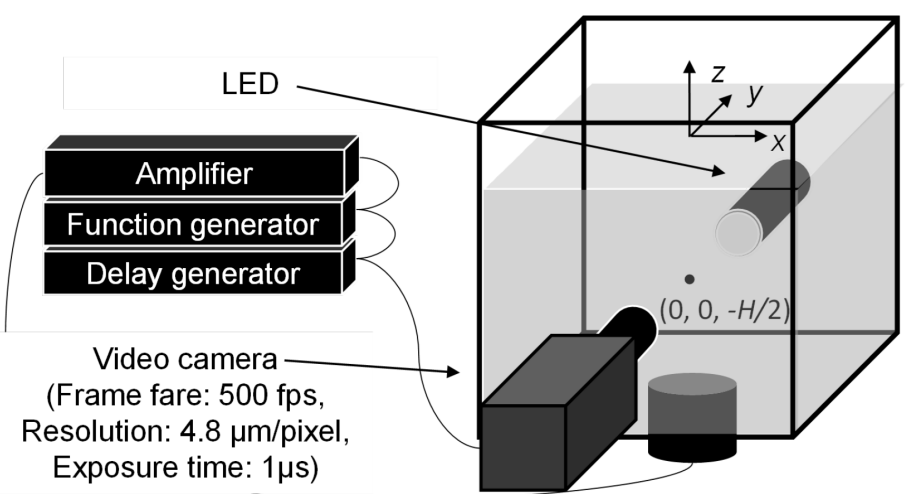

(b)

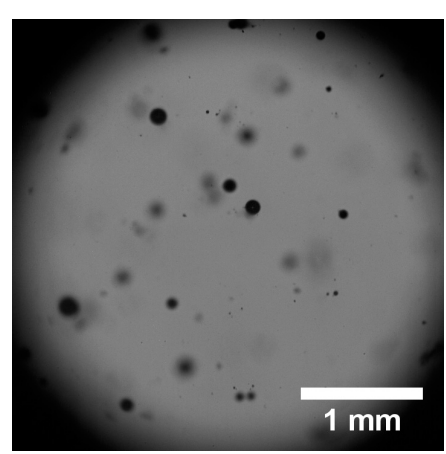

Figure 2. (a) Schematic of the setup for visualization of cavitation bubbles. (b) A typical snapshot of cavitation bubbles.

\section{Results and Discussion}

Figure 3(a,b) shows the evolution of the free-surface displacement just after the ultrasound irradiation was stopped and its frequency spectrum. Lower-order natural frequencies of the free surface are found at $4.3 \mathrm{~Hz}, 5.3 \mathrm{~Hz}, 6.2 \mathrm{~Hz}, 6.8 \mathrm{~Hz}$, and $8.0 \mathrm{~Hz}$. Figure 3(c) shows the evolution of the free-surface displacement during the ultrasound irradiation; the free surface is found to exhibit periodic oscillation. The oscillation amplitude becomes almost unchanged after $t=10 \mathrm{~s}$, meaning that the forced oscillation reaches steady state. Figure 3(d) shows the frequency spectrum of the freesurface oscillation under the steady state. There are two dominant frequency components at $4.1 \mathrm{~Hz}$ and $5.9 \mathrm{~Hz}$, which are in good agreement with the natural frequencies obtained from the free oscillation.

Figure 4(a) shows the evolution of spatially-averaged $z$-direction velocity $W(t)$ of cavitation bubbles in the visualized area. The velocity is found to change periodically about $W \approx 0$, meaning that cavitation bubbles move upward and downward periodically. Figure 4(b) shows the frequency spectrum of $W(t)$ under the steady-state oscillation of the free surface $(t=3-10 \mathrm{~s})$. There are clear peaks at $4.1 \mathrm{~Hz}$ and $5.7 \mathrm{~Hz}$, which agree with the dominant frequency components of the free-surface displacement (see Fig. 3(d)). This suggests that the up-and-down translation of cavitation bubbles are related to the free-surface oscillation. Finally, in order to investigate the spatial distribution of cavitation bubbles which is relevant to uniformity in cleaning performance, we plot the centroids of the cavitation bubbles that appeared during the steady state in Fig. 4(c). From this map, we can more clearly see stripes in the vertical direction (along the $z$ axis). These stripes are caused by the nearfield characteristics of megasonic wave transducers [9] and are found to be similar to the particle removal efficiency maps reported in [10]. However, such nonuniformity does not appear strongly in the horizontal direction. This suggests the effect of the free-surface oscillation that helps avoid the formation of steady-state, standing-wave-like pressure fields as a result of superposition between the incident and reflected waves. We may say that the free-surface oscillation can contribute to uniform cleaning particularly in the vertical direction. 
(a)

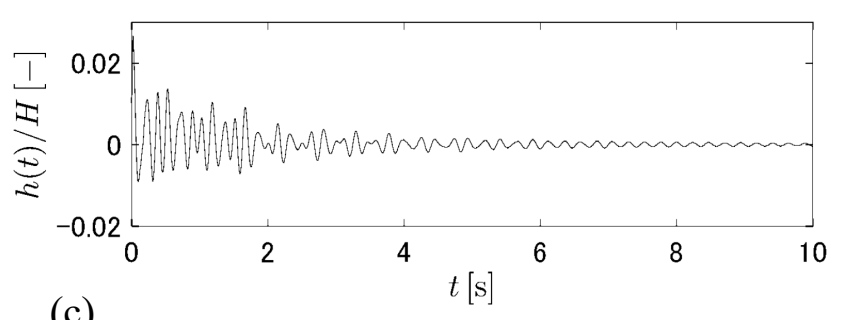

(c)

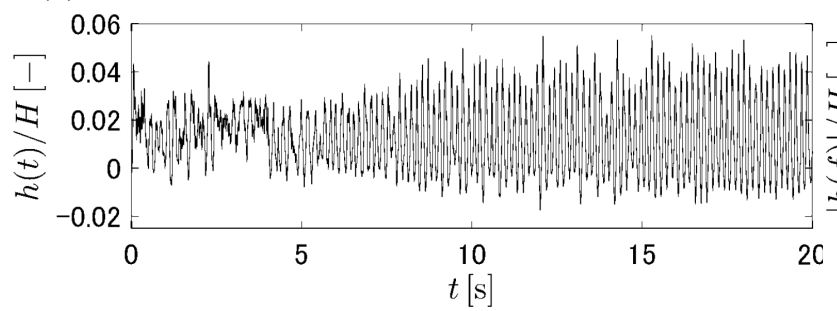

(b)

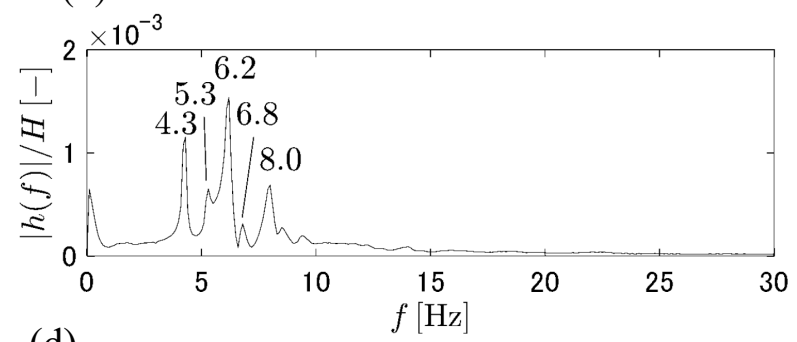

(d)

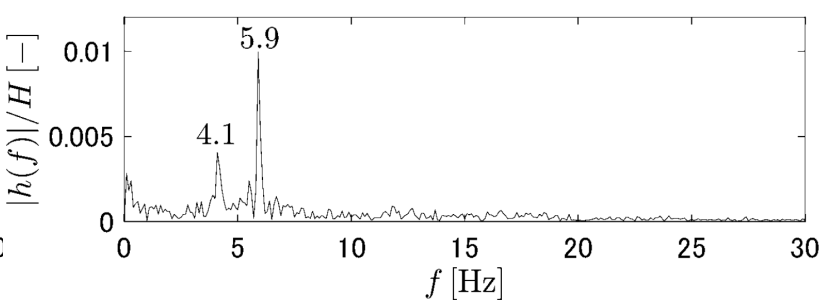

Figure 3. (a) Evolution of the free-surface displacement $h(t)$ after the ultrasound irradiation was stopped and (b) its frequency spectrum. This spectrum was obtained from the data in time interval $t=0-10 \mathrm{~s}$; (c) Evolution of the free-surface displacement $h(t)$ during the sonication and (d) its frequency spectrum. This spectrum was obtained from the data in time interval $t=10-20 \mathrm{~s}$.
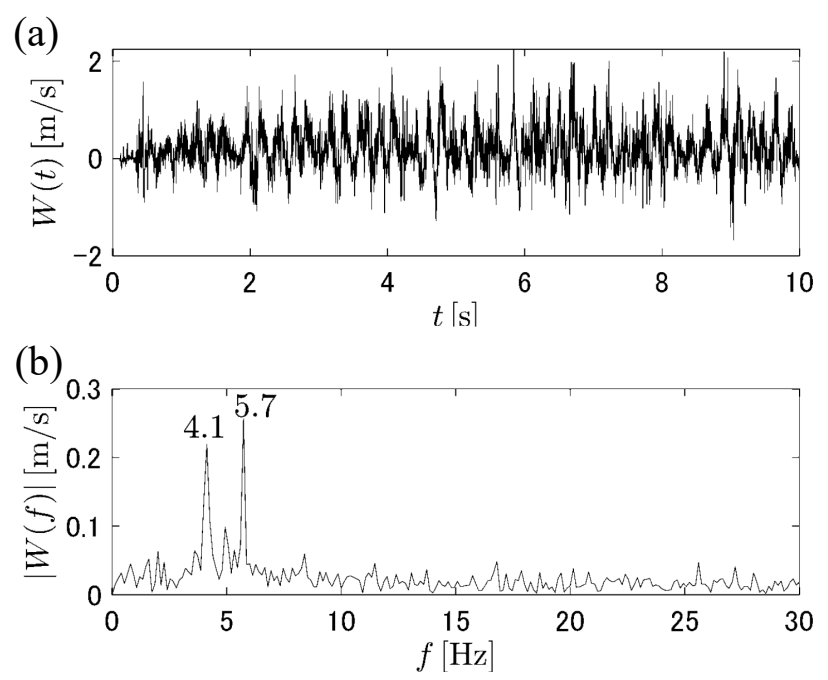

(c)

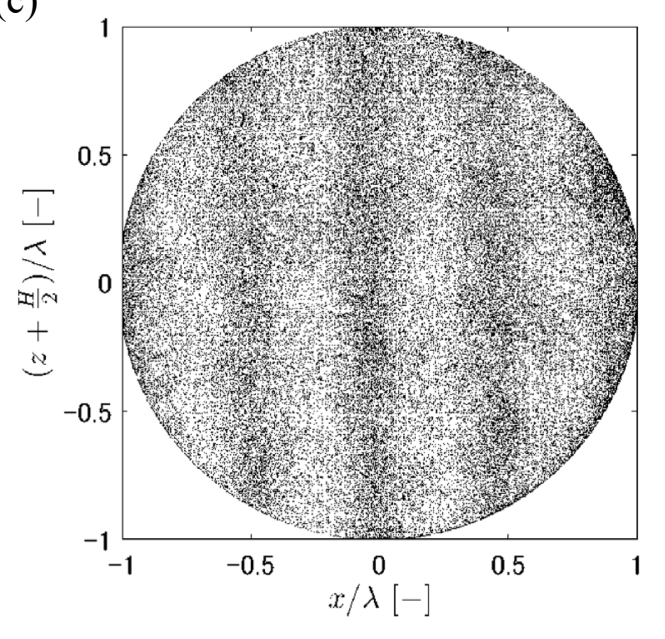

Figure 4. (a) Evolution of the spatial-averaged velocity of cavitation bubble $W(t)$ and (b) its frequency spectrum. This spectrum was obtained from the data in time interval $t=3-10 \mathrm{~s}$; (c) The centroids of the cavitation bubbles which appeared in time interval $t=3-10 \mathrm{~s}$ are plotted. The grey-scale contrast is proportional to the bubble number density; the darker region corresponds to denser bubbles.

\section{Summary}

We performed visualization of the free-surface motion and bubble translation in a $1-\mathrm{MHz}$ ultrasonic cleaning bath. It is found that the dominant frequencies of the free-surface motion under the acoustic radiation force agree with those of its natural oscillation and the up-and-down motion of cavitation bubbles, meaning that there is a strong correlation between the free-surface oscillation and bubble translation. It is found from the bubble population visualization that the free-surface oscillation is expected to contribute to uniform cleaning performance particularly in the vertical direction. 


\section{References}

[1] G.W. Gale and A.A. Busnaina: Particulate Science and Technology 13 (1995), 197.

[2] W. Kim, et al.: Applied Physics Letters, 94 (2009), 081908.

[3] L.A. Crum: Journal of the Acoustical Society of America 57 (1975), 1363.

[4] T. Yamashita and K. Ando: Ultrasonics Sonochemistry 52 (2019), 268.

[5] T. Kamakura, et al.: Nonlinear Acoustics (CORONA PUBLISHING CO., LRD, Japan, 2014).

[6] K. Tsuchiya, et al.: Aerosol Research 26 (2011), 11.

[7] F.J. Fahy: Sound Intensity (Elsevier Science Pub. Co., USA, 1989).

[8] M. Minnaert: Philosophical Magazine, 16 (1933), 235.

[9] D.T. Blackstock: Fundamentals of Physical Acoustics (Wiley-Interscience, USA, 2000).

[10] G. Vereecke, et al.: Ssolid State Phenomena, 92 (2003), 143. 